\title{
Disección espontánea de arteria ilíaca asociada a ejercicio y uso de ergotamina*
}

\author{
Drs. JUAN PABLO FUENZALIDA G. ${ }^{1}$, ANGEL PUENTES R. ${ }^{1}$, SEBASTIÁN TERRAZAS F. ${ }^{2}$, \\ SANDRA OSORIO V. ${ }^{1}$, JORGE VERGARA C. ${ }^{1}$, EITAN SCHWARTZ Y. ${ }^{1}$, RUBÉN SOUMASTRE CH. ${ }^{1}$
}

Departamento de Enfermedades Cardiovasculares.

2 Servicio de Urgencia Adultos.

Clínica Dávila, Santiago, Chile.

\begin{abstract}
Spontaneous dissection of iliac artery related with excercise and ergotamine. A case report

Introduction: Spontaneous dissection of the iliac artery (SDIA) is an extremely rare clinical manifestation, associated to different etiologies, and it usually shows an ischemia of the involved lower extremity. Clinical case: We report a case of a 48-year-old man, with past medical history of chronicle consumption of ergotamine, which presented left lower limb ischemia, while practicing physical exercise. An emergent contrast-enhanced computed tomography scan showed a spontaneous dissection of the common and the external left iliac artery. An endovascular therapy of the lesion was performed with self-expanding stents, achieving the reconstruction of the lesion, the recovery of the blood flow and of the lower limb ischemia. Conclusions: There are no previous descriptions of the association between ergotamine consumption, sport and this very rare pathology. Endovascular treatment represents a less invasive and, such as in our case report, successful management of the SDIA, and it should be considered among the alternative therapies.
\end{abstract}

Key words: Iliac artery, dissection, stent, sport, ergotamine.

\section{Resumen}

Introducción: La disección espontánea de la arteria ilíaca es un cuadro muy poco frecuente asociado a diferentes etiologías que habitualmente se presenta como isquemia de la extremidad comprometida. Caso clínico: Se reporta el caso de un paciente masculino de 48 años con antecedente de consumo crónico de ergotamina, quien mientras practicaba deporte presenta cuadro de isquemia aguda de la extremidad inferior izquierda. Angio tomografía computada demostró disección espontánea de la arteria ilíaca común y externa izquierda. Se realizó terapia endovascular de la lesión con stents auto expandibles, logrando la reparación de la lesión, el restablecimiento del flujo y la recuperación de la isquemia de la extremidad. Discusión y conclusiones: No existen reportes previos de esta patología poco frecuente, en que se asocie en forma conjunta la práctica de deporte y el uso de ergotamina. Dentro de las alternativas terapéuticas, la reparación endovascular representa una opción menos invasiva y, como en este caso, con óptimos resultados.

Palabras clave: Arteria ilíaca, disección, stent, ejercicio, ergotamina.

*Recibido el 22 de mayo de 2012 y aceptado para publicación el 11 de junio de 2012.

Los autores no refieren conflictos de interés.

Correspondencia: Dr. Juan Pablo Fuenzalida G.

Av. Recoleta 464. Santiago, Chile. CP: 8431657

fuenzalidajp@gmail.com 


\section{Introducción}

La disección espontánea de la arteria ilíaca es un cuadro poco frecuente que puede presentarse en asociación a diferentes etiologías y que habitualmente se presenta con signos y síntomas de isquemia de la extremidad comprometida. Se presenta el caso de un paciente con esta patología, asociada al ejercicio y consumo de ergotamina, que fue resuelta por vía endovascular.

\section{Caso clínico}

Paciente de 48 años, de sexo masculino, sin antecedentes móbidos ni factores de riesgo cardiovascular, excepto por el consumo crónico de ergotamina por migraña ( 3 a 5 mgr a la semana durante aproximadamente 10 años). Jugador habitual de tenis, deporte que practicaba regularmente unas tres veces por semana. Mientras jugaba tenis, presenta cuadro de inicio brusco con dolor intenso en región inguinal izquierda más parestesias y frialdad en muslo, pierna y pie ipsilateral. Consulta en Servicio de Urgencia donde se evidencia paciente normotenso, sin taquicardia, con dolor a la palpación en fosa ilíaca y región inguinal izquierda, sin soplos, con palidez y disminución de temperatura en toda la extremidad inferior izquierda y ausencia de pulso femoral, poplíteo, tibial posterior y pedio de ese lado. Se realiza angio tomografía computarizada (angioTC) que demuestra disección de arteria ilíaca común y externa izquierda, sin otros hallazgos patológicos de enfermedad arterial, sin signos de fibrodisplasia (Figuras 1, 2 y 3). Se efectúa reparación endovascular de la lesión con dos stents auto

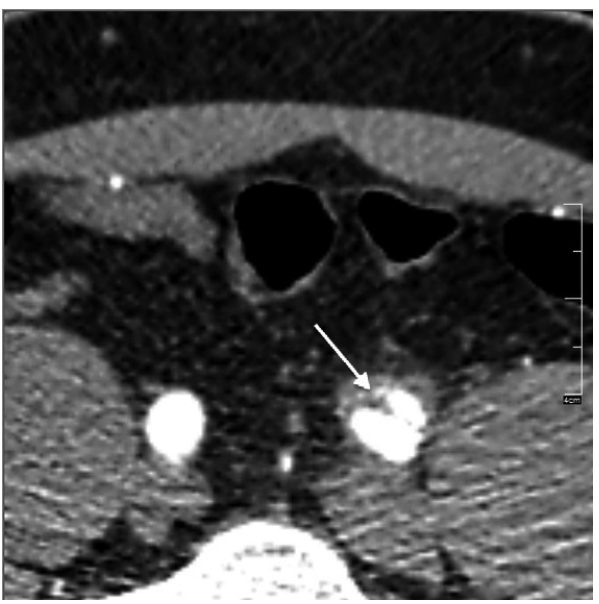

Figura 1. Corte axial de angioTC que muestra zona de disección de arteria ilíaca común izquierda. expandibles (Zilver ${ }^{\circledR}$ Cook Medical ${ }^{\circledR}$ ) de 14 x 60 mm y 12 x $60 \mathrm{~mm}$ en arteria ilíaca común y externa izquierda, lográndose restablecimiento del flujo y recuperación de isquemia de la extremidad, con reaparición de todos los pulsos (Figura 4). Es dado de alta a los 3 días postoperatorio con indicación de antiagregación plaquetaria con ácido acetilsalicílico en dosis de 100 mgr al día. Es controlado en forma ambulatoria al mes, 3 y 6 meses postoperatorios, encontrándose asintomático. Se realizó angioTC de control al mes postoperatorio que demuestra permeabilidad de stents a nivel de arterias ilíaca común y externa, así como de la arteria ilíaca interna (Figura 5). Estudio vascular no invasivo muestra curvas

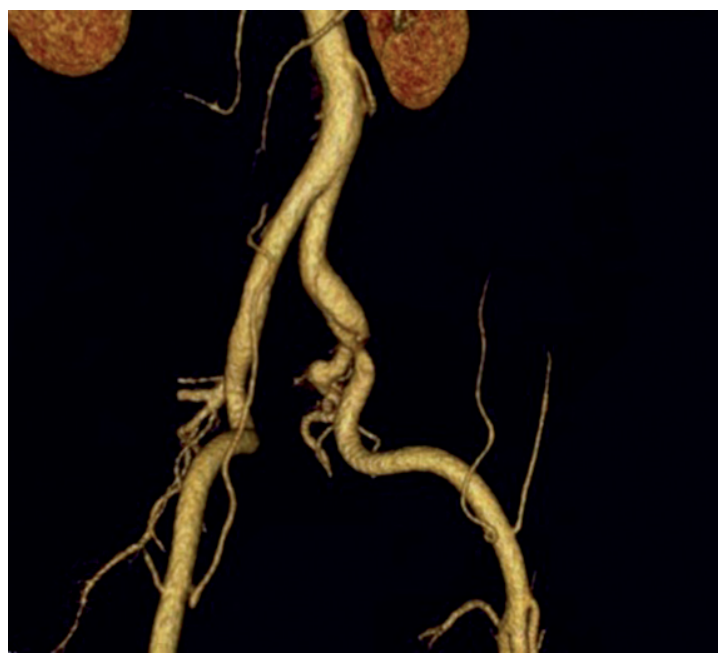

Figura 2. Reconstrucción tridimensional en la que se observa zona de disección de arteria ilíaca común y externa izquierda.

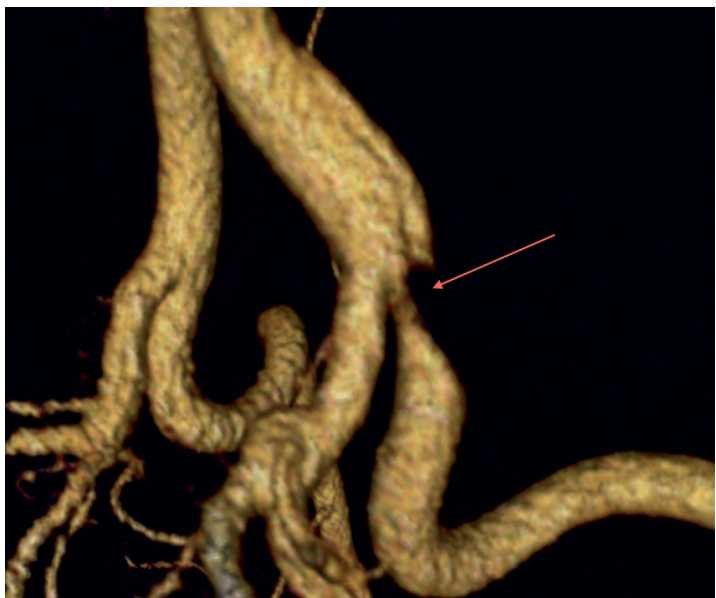

Figura 3. Ampliación que evidencia zona de paso filiforme en origen de arteria ilíaca externa. 


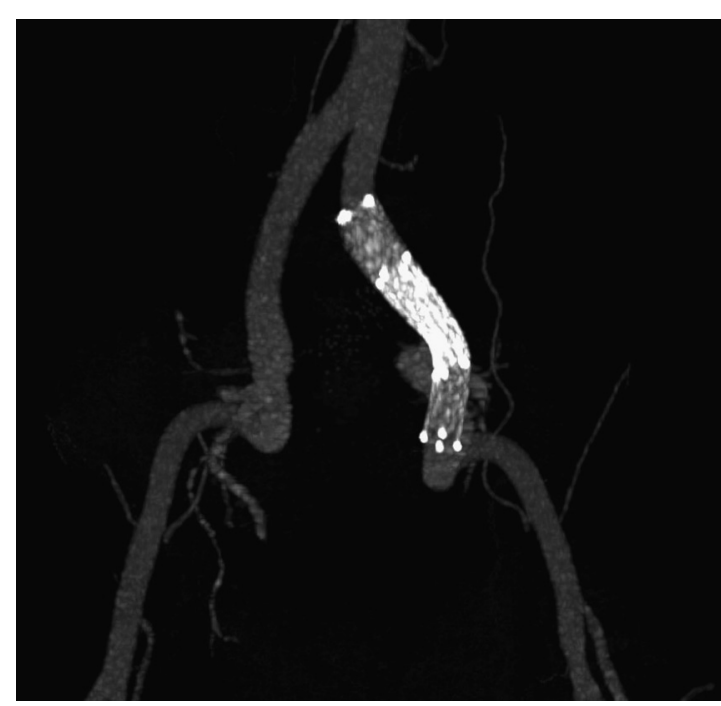

Figura 4. Imagen de reparación endovascular con stents autoexpandibles.

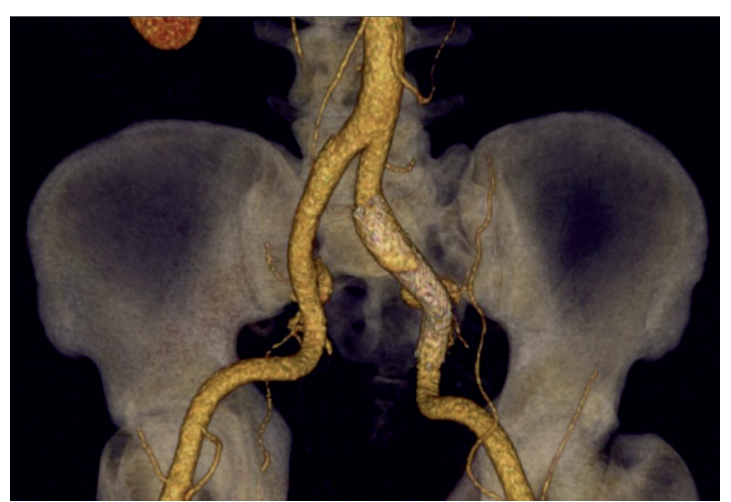

Figura 5. Reconstrucción tridimensional que muestra permeabilidad de arterias ilíaca común, interna y externa.

de pletismografía, presiones segmentarias e índices tobillo-brazo en rangos normales. Se mantiene con indicación de antiagregación, mantener suspensión de derivados de ergotamina y con la restricción de realizar deporte sólo en forma moderada.

\section{Discusión}

La disección espontánea de las arterias ilíacas, sin compromiso de la aorta, es un cuadro muy poco frecuente. En una revisión de 1966 a 2006 se reportan sólo 34 casos publicados ${ }^{1}$. Esta entidad, se ha asociado a diferentes etiologías, entre ellas, embarazo, aterosclerosis, fibrodisplasia, síndrome de Marfán y ejercicio ${ }^{1-4}$. En la literatura sólo existe un reporte que relaciona esta entidad al uso crónico de ergotamina, tratándose este caso de una mujer que desarrolló simultáneamente disección de ambas arterias ilíacas externas, sin existir claridad completa respecto al rol de la ergotamina en el desarrollo de esta patología 5 . Se ha descrito su aparición en deportistas altamente entrenados, en los cuales existirían cambios hemodinámicos locales asociados al origen de este cuadro ${ }^{2}$.

Esta entidad clínica habitualmente se manifiesta por dolor en la región inguinal del lado involucrado, con dos formas de presentación: algunos pacientes que cursan con claudicación progresiva de días o meses de evolución o, como en nuestro caso, con isquemia aguda de la extremidad comprometida. Habitualmente se compromete una sola extremidad, pero existen reportes de compromiso bilateral ${ }^{5-7}$, generalmente se presenta en mayores de 40 años, especialmente en aquellos casos relacionados con ejercicio $^{2,8,9}$.

Dentro de los métodos diagnósticos se encuentran la ecotomografía Doppler, la cual permitiría ver la zona de disección y los cambios hemodinámicos asociados a ella, con disminución del flujo arterial distal, pero es la angiotomografía computarizada la que nos aporta mayor información respecto a la confirmación del diagnóstico, localización y extensión de la lesión y la posible coexistencia de otras lesiones arteriales que orienten a la etiología del cuadro, así como permite la planificación de la terapia.

Respecto a las opciones terapéuticas, desde las primeras descripciones de casos de esta entidad, se han planteado el manejo conservador ${ }^{6}$, la reconstrucción quirúrgica ${ }^{2,4,8,10,11}$ y la reparación endovascular con stents ${ }^{5,11-13}$, siendo actualmente esta terapia de elección por representar una alternativa menos invasiva y con muy buenos resultados, como en el caso de este paciente.

\section{Conclusión}

La disección espontánea de arterias ilíacas es una entidad poco frecuente, asociada a diferentes etiologías.

No existe reporte previo en la literatura que asocie ejercicio y uso de ergotamina dentro de las etiologías de este cuadro.

La terapia endovascular representó, en este caso, una óptima alternativa de tratamiento.

\section{Referencias}

1. Honjo O, Sano S. Spontaneous Isolated Dissection of Iliac Artery: Unique Vascular Event Resulting from Various Etiologies. Vascular Disease Prevention 2006;3:391-9. 
2. Cook PS, Erdoes LS, Selzer PM, Rivera FJ, Palmaz JC. Dissection of the external iliac artery in highly trained athletes. J Vasc Surg. 1995;22:173-7.

3. Luck I, Hanschke D, Geissler, C. Spontaneous dissection of external iliac artery due to fibromuscular dysplasia. Vasa 2002;31:115-21.

4. Akashi H, Nata S, Kanaya K, Shintani Y, Onitsuka S, Aoyagi S. Spontaneous dissection of the iliac artery in a patient with fibromuscular displasia. Ann Vasc Surg. 2010; 24:952.e13-952.e16.

5. Molkara AM Abou-Zamzam AM JR, Teruya TH Binchi C, Killeen JD. Chronic ergot toxicity presenting with bilateral external iliac artery dissection and lower extremity rest pain. Ann Vasc Surg, 2006;20:803-8.

6. Fukui S, Chelbi E, Paraskevas N, Soury P, Gigou F, Petit MD, Laurian C. Bilateral dissection of external iliac artery. Ann Vasc Surg 2007;21:373-5.

7. Engin C, Calkavur T, Apaydin AZ, Durmaz I. Bilateral Spontaneous and Isolated Dissection of the External Iliac Arteries: Report of a Case. Eur J Vasc Endovas Surg. 2005;9:19-21.
8. Savolainen H, Heller G, Fleischmann A, Widmer MK, Carrel Tp, Schmidli J. Spontaneous dissection of common iliac artery: A case report. Vasc Endovasc Surg. 2004;38:263-5.

9. Kwon SH, Oh JH. Successful interventional treatment of a spontaneous right common iliac artery dissection extending retrogradely into the left external iliac artery] 2006;17:717-21.

10. Declemy S, Kreitmann A, Popoff G, Diaz A. Spontaneous dissecting aneurysm of the common iliac artery 1991;5:549-51.

11. Fernández AL, Herreros JM. Spontaneous and isolated dissection of the common iliac artery. J Cardiovasc Surg. (Torino) 1997;38:377-9.

12. Kwak HS, Han YM, Chung GH, Yu HC, Jeong YJ. Isolated Spontaneous Dissection of the Common Iliac Artery: Percutaneous Stent Placement in Two Patients. Card Vasc and Int Rad. 2006:29:883-5.

13. Teh LG, Sieunarine K, Van Schie G, Vasudevan T, Spontaneous common iliac artery dissection after exercise. J Endovasc Ther. 2003;10:163-6. 